\title{
Anti-lysobisphosphatidic acid antibodies in patients with antiphospholipid syndrome and systemic lupus erythematosus
}

C. Alessandri, ${ }^{\dagger}$ M. Bombardieri, ${ }^{\dagger}$

L. Di Prospero, ${ }^{\star}$ P. Conigliaro, F. Conti,

G. Labbadia, R. Misasi, ${ }^{\star}$ M. Sorice ${ }^{\star}$ and

G. Valesini

Cattedra Di Reumatologia, Dipartimento di

Clinica e Terapia Medica Applicata, Università ' $\mathrm{La}$

Sapienza', Rome, Italy, and ${ }^{\star}$ Dipartimento di

Medicina Sperimentale e Patologia, Università ' $\mathrm{La}$

Sapienza', Rome, Italy

Accepted for publication 2 December 2004

Correspondence: Professor Guido Valesini,

Dipartimento di Clinica e Terapia Medica Appli-

cata, Cattedra di Reumatologia, Università 'La

Sapienza', V. le del Policlinico 155, 00161 Rome, Italy.

E-mail: guido.valesini@uniroma1.it

$\dagger$ These authors contributed equally to this study.

\begin{abstract}
Summary
Lyso(bis)phosphatidic acid (LBPA) is a novel antigenic target in antiphospholipid syndrome (APS) and antibodies directed against LBPA (aLBPA) have been detected in sera from APS patients. In this study we first evaluated aLBPA in comparison with the most widely used methods (i.e. anticardiolipin [(aCL)-enzyme-linked immunosorbent assay (ELISA)] and antibeta-2glycoprotein-I antibodies ( $\boldsymbol{\beta}_{2}$-GPI-ELISA) utilized to detect antiphospholipid antibodies in patients with primary or secondary APS, systemic lupus erythematosus, chronic HCV infection and healthy subjects. We then assessed the relationship between aLBPA, lupus anticoagulant (LAC) and the main clinical manifestations of APS. Finally, we evaluated the presence of 'pure' (i.e. $\beta_{2}$-GPI-independent) aLBPA in patients with APS and controls. The results indicate that aLBPA as well as a $\boldsymbol{\beta}_{2}$-GPI display higher specificity but lower sensitivity for APS compared to aCL. Moreover, serum aLBPA correlate closely with $\mathrm{aCL}$ and $\mathrm{a} \boldsymbol{\beta}_{2}$-GPI in APS patients and are strictly associated with LAC positivity. We demonstrate that $\boldsymbol{\beta}_{2}$-GPI binds to LBPA with affinity similar to $\mathrm{CL}$, and antibodies able to react with phosholipid-protein complex exist; however, 'pure' aLBPA can also be detected in sera of APS patients. Altogether these data confirm that LBPA may be an antigenic target in APS and that aLBPA are serological markers of APS with similar sensitivity and specificity compared to a $\beta_{2}$-GPI. However, the clinical utility of aLBPA detection alone or in combination with $\mathrm{aCL}$ and/or $\mathrm{a}_{2}$-GPI remains to be elucidated in larger and longitudinal studies.
\end{abstract}

Keywords: antiphosholipid syndrome, autoantibodies, cardiolipin, lyso(bis)phosphatidic acid, systemic lupus erythematosus

\section{Introduction}

Antiphospholipid syndrome (APS) is a disease characterized by arterial and venous thrombosis, recurrent miscarriages or fetal loss and circulating antiphospholipid antibodies (aPL) directed against different anionic phospholipids, mainly cardiolipin (CL) [1,2]. In the last two decades several reports have indicated that the main antigenic target of these autoantibodies are phospholipid binding proteins, most notably beta-2-glycoprotein I ( $\beta_{2}$-GPI) [3-5], or a complex of these proteins with phospholipids [6-9]. Nevertheless, in this context several contradictory results have been reported and the exact nature of the aPL antigens is still a matter of controversy [3-10]. Recently, it has been described that a phospholipid restricted to the late endosome, the lyso(bis)phosphatidic acid (LBPA), could represent a novel antigenic target in the course of APS [11].

LBPA is a hydrophobic isomer of phosphatidylglycerol, related structurally to cardiolipin, localized in specialized domains within the internal membranes of multi-vesicular late endosomes. These organelles are constituted by a limiting membrane and an internal membrane, the latter containing triglycerides, cholesterol esters and a subset of phospholipids forming particular domains involved in protein-sorting steps [11-13]. Interestingly, human sera from patients with APS label late endosomes containing LBPA and induce a redistribution of intracellular $\beta_{2}$-GPI, a protein involved in the pathogenesis of APS [14]. Moreover, we have reported that anti-LBPA antibodies (aLBPA) are able to exert lupus anticoagulant activity in vitro, supporting the view 
that aLBPA may have a pathogenic role in APS [14]. Nevertheless, a few studies, restricted to a low number of patients, focused on the association between the presence of aLBPA and clinical manifestations in APS patients [11,14,15].

In the present study we evaluated serum aLBPA in patients with primary or secondary APS, systemic lupus erythematosus (SLE), chronic HCV infection and healthy controls. The serum levels of aLBPA were correlated to the clinical manifestations and compared to the levels of anti-CL antibodies $(\mathrm{aCL})$ and anti $\beta_{2}$-GPI antibodies $\left(\mathrm{a} \beta_{2}\right.$-GPI) in all patient groups.

\section{Patients, materials and methods}

\section{Subjects}

Seventy-three consecutive out-patients, attending the Rheumatology Division of the University of Rome 'La Sapienza', were enrolled. Thirty patients had APS, diagnosed according to the Sapporo criteria [2], primary $(N=15)$ or secondary $(N=15)$ to other diseases (13 SLE, one sarcoidosis, one mixed connective tissue disease); 43 patients had SLE fulfilling the ACR revised criteria for the classification of SLE [16]. We also enrolled 37 patients with chronic HCV infection and 40 healthy subjects (normal blood donors) matched for age and sex as controls.

After informed consent was obtained, each subject underwent peripheral blood sample collection. The serum recovered was then stored at $-20^{\circ} \mathrm{C}$ until assayed.

\section{Materials}

CL (bovine heart) was obtained from Sigma Chemical Co. (St Louis, MO, USA). LBPA and hydrocardiolipin (HCL) were obtained from Avanti Polar Lipids (Alabaster, AL, USA). High performance thin layer chromatography (HPTLC) was performed as reported previously [17] to assess the presence of cross-contamination between phospholipid preparations. Human $\beta_{2}$-GPI was obtained from Chemicon International (Temecula, CA, USA).

The following antibodies were used: rabbit polyclonal antihuman $\beta_{2}$-GPI (Chemicon International); goat polyclonal antihuman IgG, IgA, IgM alkaline phosphatase conjugate (Sigma); and mouse antirabbit IgG alkaline phosphatase conjugate (Sigma).

Human IgG fractions were first isolated with 33\% ammonium sulphate fractionation from plasma of patients with APS and from healthy donors; the enriched fractions were then centrifuged at 10000 r.p.m. for $30 \mathrm{~min}$ and resuspended in one-fourth of the original volume of distilled water. Samples were dialysed overnight against $0.01 \mathrm{M}$ ammonium carbonate, and then separated using a Progel TSK G3000 column (Supelco, Bellefonte, PA, USA). IgG fractions were obtained eluted with $0 \cdot 2 \mathrm{M}$ phosphate buffer and subsequently dialysed against 51 of distilled water. Pro- tein concentration was measured with the Lowry method [18] and the purity of the IgG preparations was checked by sodium dodecyl sulphate-polyacrylamide gel electrophoresis (SDS-PAGE).

\section{Enzyme-linked immunosorbent assay (ELISA) for aLBPA, aCL and anti $\boldsymbol{\beta}_{2}$-GPI IgG}

Serum aLBPA and aCL were detected by means of an ELISA. Phospholipids at $50 \mu \mathrm{g} / \mathrm{ml}$ concentration in ethanol were used to coat microtitre plates overnight at $4^{\circ} \mathrm{C}$. After four washes with phosphate buffered saline (PBS), plates were blocked for $1 \mathrm{~h}$ at room temperature (RT) with $10 \%$ fetal calf serum (FCS) in PBS (PBS-F) or $0 \cdot 25 \%$ gelatine (PBS-G) to assess the binding to LBPA and CL in the absence of $\beta_{2}$-GPI in indicated experiments. After four washes with PBS-F (or PBS-G), plates were incubated for $90 \mathrm{~min}$ at RT with sera diluted at $1: 50$ or human IgG $(100 \mu$ l of concentrated solutions of $4.8 \mathrm{mg} / \mathrm{ml}$ ) in PBS-F (or PBS-G). Subsequently, after four washes, plates were incubated for $90 \mathrm{~min}$ at RT with goat polyclonal antihuman IgG-IgA-IgM alkaline phosphatase conjugated antibodies (Sigma) diluted at $1: 1000$ in PBS-F (or PBS-G). After four washes, a solution of paranitrophenyl phosphate tablets in ethanolamine was used for the enzyme reaction and the plates were read at a $405 \mathrm{~nm}$ wavelength. All assays were performed in duplicate and the absorbance of control wells was subtracted to account for non-specific binding. A titration curve of two positive reference sera (with medium-high ELISA immunoreactivity for aLBPA and aCL, respectively) was performed to show the performance of the tests.

In order to investigate the specificity of the assay, absorption tests were performed according to techniques described elsewhere $[19,20]$. Briefly, three aLBPA/aCL-positive sera (1/ 50 in $1 \%$ PBS-BSA) were preincubated for $60 \mathrm{~min}$ at $37^{\circ} \mathrm{C}$ with increasing amounts of LBPA and CL dried onto the surface of glass tubes. Subsequently, after a centrifugation step (10000 $g$ for $30 \mathrm{~min}$ ) the supernatants were tested for autoantibody reactivity, as above. As control, in these experiments we further used a fully hydrogenated CL derivative (HCL) that does not occur naturally, but can provide a reference structure for basal immunorecognition of the lipid [20].

Intra- and interassay variations were determined by assaying two samples (one APS patient and one SLE patient) 16 times in one assay (intra-assay) and assaying the two samples in quadruplicate in nine consecutive assays (interassay). Finally, to establish the clinical significance of aLBPA and aCL we considered these autoantibodies as positive when the OD was higher than the cut-off value corresponding to the mean plus 5 s.d. of 40 healthy controls matched for sex and age.

Anti- $\beta_{2}$-GPI IgG ELISA kits were obtained from Diamedix (Miami, FL, USA) and performed according to the manufacturer's instructions. To evaluate the binding of $\beta_{2}$-GPI to 
LBPA and CL, plates were coated overnight with an increasing amount of these lipids and then reacted with PBS-F, containing an excess of $\beta_{2}$-GPI, for $2 \mathrm{~h}$ at RT. A rabbit polyclonal anti $\beta_{2}$-GPI was used to detect the binding of $\beta_{2}$-GPI to lipids.

\section{Immunostaining on TLC plates for aLBPA and aCL}

The immunostaining of TLC plates (Merck, Darmstadt, Germany) was performed as described previously [21], using $2.5 \mu \mathrm{g}$ of LBPA or CL. Affinity-purified IgG fractions $(2.4 \mathrm{mg} / \mathrm{ml})$ from APS sera (which had been deemed aLBPA and aCL-positive by ELISA screening) and normal human sera were diluted $1: 100$ in PBS containing 0.5\% (w/v) gelatine. Parallel blots were processed without primary antibody or without antigen as control for non-specific reactivity.

\section{Statistical analysis}

Statistical analysis was carried out using the Mann-Whitney $U$-test for comparison of means between different groups of patients and controls. Wilcoxon's paired test was used to compare differences between autoantibody profiles in each group. Qualitative differences between subgroups were analysed by $\chi^{2}$ or Fisher's exact test when appropriate. Correlation analysis was carried out by the Spearman test. A $P$-value $<0.05$ was considered statistically significant.

\section{Results}

\section{Patients clinical details}

The demographic and clinical features of the patients and controls enrolled in this study are reported in Table 1. SLE patients were used as diseased controls compared to APS (primary or secondary). All SLE patients were consecutive out-patients attending the rheumatology clinic and met the ACR criteria for SLE. None of the 43 patients in the SLE without APS group fulfilled the Sapporo criteria for APS. None of the healthy subjects experienced arterial or venous thrombosis, nor recurrent fetal loss. The lupus anticoagulant (LAC) positivity was deduced in each case from recent clinical records and measured following the guidelines proposed by the Subcommittee for Standardization of Lupus Anticoagulants (SSC) of the Scientific and Standardization Committee of the ISTH [22]. In particular, at our institution LAC was determined by kaolin-clotting time, the textarin time, a modified dilute Russell's viper venom time (DRVVT) and confirmed by the Staclot LA test.

\section{aLBPA and aCL ELISA}

To compare immunoreactivity of LBPA and CL, it was important to ascertain that the lipid preparations were not cross-contaminated. By means of HPTLC, we could not observe any detectable contamination in lipid preparations (data not shown).

Serum levels of aLBPA and aCL IgG in patients and controls were quantified by ELISA (Fig. 1, Table 2). Similar results were obtained in selected patients when purified IgGs instead of sera were used (data not shown). In the present study immunoreactivity to LBPA and CL was significantly higher in APS and SLE patients with respect to controls (see Fig. 1 and Table 2 for statistical significance). According to our cut-off (mean plus 5 s.d. of 40 normal controls) the prevalence of aLBPA IgG was 53.3\% (16 of 30) in APS, $4 \cdot 6 \%$ (two of 43 ) in SLE and $2 \cdot 7 \%$ (one of 37 ) in HCV patients, sensitivity (95\% CI) $0.53(0.45-0.61)$, specificity (95\% CI) 0.97 (0.95-1.00). The prevalence of aCL IgG was $80 \%$ (24 of 30 ) in APS, $16 \cdot 3 \%$ (seven of 43 ) in SLE and $8 \cdot 1 \%$ (three of 37 ) in HCV patients, sensitivity (95\% CI) $0 \cdot 8(0 \cdot 73-0 \cdot 86)$, specificity (95\% CI) $0.92(0.89-0.94)$. In Table 2 the isotype distribution of aLBPA and aCL-positive sera in patients groups is reported. Finally, no difference in immunoreactivity to LBPA (as well as CL and $\beta_{2}$-GPI) was observed in APS patients divided into primary and secondary APS. This, together with

Table 1. Demographic and clinical data of patients studied

\begin{tabular}{lcccc}
\hline Variable & $\begin{array}{c}\text { APS } \\
(N=30)\end{array}$ & $\begin{array}{c}\text { SLE } \\
(N=43)\end{array}$ & $\begin{array}{c}\text { HCV } \\
(N=37)\end{array}$ & $\begin{array}{c}\text { NHS } \\
(N=40)\end{array}$ \\
\hline $\begin{array}{l}\text { Females/males } \\
\text { Ethnicities }\end{array}$ & $\begin{array}{c}23 / 7 \\
\text { Caucasian }\end{array}$ & $\begin{array}{c}38 / 5 \\
\text { Caucasian }\end{array}$ & $\begin{array}{c}14 / 23 \\
\text { Caucasian }\end{array}$ & $\begin{array}{c}22 / 18 \\
\text { Caucasian }\end{array}$ \\
$\begin{array}{l}\text { Mean (range) } \\
\text { Disease duration, months }\end{array}$ & $34(7-68)$ & $36 \cdot 8(14-70)$ & $55 \cdot 4(20-74)$ & $53(32-70)$ \\
Mean (range) & $143(1-322)$ & $107(6-312)$ & $41 \cdot 2(3-132)$ & 0 \\
Venous thromboses (\%) & $18(60)$ & $2(4 \cdot 6)$ & 0 & 0 \\
Arterial thromboses (\%) & $13(43 \cdot 3)$ & 0 & 0 & 0 \\
Recurrent thromboses (\%) & $11(36 \cdot 6)$ & 0 & 0 & 0 \\
Miscarriages/pregnancy (\%) & $11 / 14(78 \cdot 5)$ & $5 / 14(35 \cdot 7)$ & $2 / 11(18 \cdot 1)$ & 0 \\
Lupus anticoagulant activity (\%) & $21(70)$ & $10(23 \cdot 2)$ & n.d. & n.d. \\
\hline
\end{tabular}

n.d. $=$ not done. 
Fig. 1. ELISA for aLBPA aCL and a $\beta_{2}$-GPI IgG. Box-and-whisker plot of IgG autoantibodies binding to CL and LBPA detected by means of an ELISA in APS patients ( $n=15$ primary and 15 secondary to other diseases), SLE patients ( $n=43)$, patients with HCV $(n=37)$ and normal controls $(n=40)$. Median, quartiles, range and possibly extreme values are shown. $\dagger$, APS versus SLE: $P=0 \cdot 0000$; , APS versus HCV: $P=0 \cdot 0000$; \#, APS versus NHS: $P=0 \cdot 0000$; ${ }^{\star}$, SLE versus HCV: $P=\mathrm{NS} ; \Phi$, SLE versus NHS: $P=0.0000$; HCV versus NHS: $P=0.0000$; , APS versus SLE: $P=0 \cdot 0000$; $\mathbf{\square}$, APS versus HCV: $P=0 \cdot 0000$; , SLE versus HCV: $P=\mathrm{NS} ; \varphi$, SLE versus NHS: $P=0 \cdot 0000 ; \$, \mathrm{HCV}$ versus NHS: $P=0 \cdot 0000$.
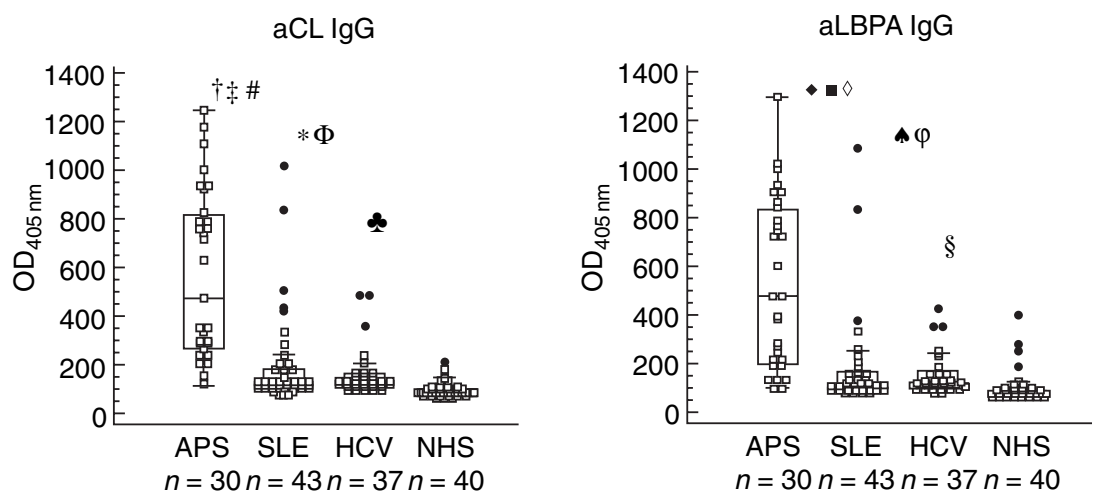

the very low prevalence of anti-LBPA in patients with SLE without APS, confirms that anti-LBPA are closely associated with APS independently in the presence of an underlying connective tissue disease.

The specificity of the assays was examined by dilution and absorption experiments. Serial dilutions of positive aLBPA and aCL sera showed a progressive reduction of the antibodies binding, while this reactivity was enhanced as concentration of lipids on coated wells was raised from 1 to $50 \mu \mathrm{g} / \mathrm{ml}$ (Fig. 2a). Conversely, binding of both positive sera was significantly inhibited by preincubation with increasing amounts of LBPA and CL, respectively (Fig. 2b). None of the sera showed reactivity to HCL and absorption with HCL did not influence aLBPA and aCL reactivity. Finally, the coefficients of intra-assay and interassay variation of the two samples assayed were $4 \cdot 6 \%, 5 \cdot 2 \%$ and $12 \cdot 6 \%, 13 \cdot 6 \%$, respectively.

To establish the clinical significance of aLBPA we grouped APS patients according to serological and clinical findings. As expected, we found a significantly increased prevalence of $\mathrm{aCL}$ and $\mathrm{a} \beta_{2}$-GPI IgG in patients who presented LAC posi-

Table 2. IgG, IgM and IgA isotype distribution of aCL and aLBPA in the different populations studied; the numbers represent number of patients with percentages in brackets

\begin{tabular}{lccl}
\hline & APS $(n=30)$ & SLE $(n=43)$ & HCV $(n=37)$ \\
\hline aCL $(\%)$ & & & \\
IgG & $24(80)$ & $7(16 \cdot 2)$ & $3(8 \cdot 1)$ \\
IgM & $14(46 \cdot 6)$ & $3(6 \cdot 9)$ & $4(10 \cdot 8)$ \\
IgA & $4(13 \cdot 3)$ & $2(4 \cdot 6)$ & $1(2 \cdot 7)$ \\
Total (antihuman Ig) & $24(80)$ & $10(23 \cdot 2)$ & $7(18 \cdot 9)$ \\
aLBPA (\%) & & & \\
IgG & $16(53 \cdot 3)$ & $2(4 \cdot 6)$ & $1(2 \cdot 7)$ \\
IgM & $8(26 \cdot 6)$ & $1(2 \cdot 3)$ & $3(8 \cdot 1)$ \\
IgA & $7(23 \cdot 3)$ & $2(4 \cdot 6)$ & $0(0)$ \\
Total (antihuman Ig) & $19(63 \cdot 3)$ & $3(6 \cdot 9)$ & $4(10 \cdot 8)$ \\
\hline
\end{tabular}

(a) Dilution assay

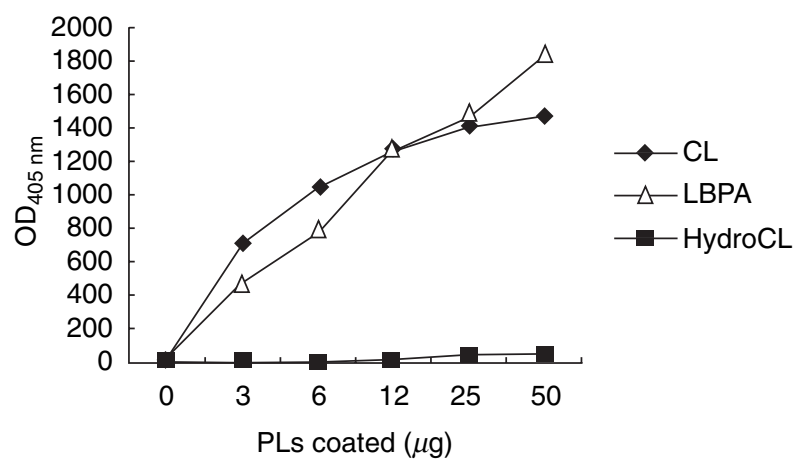

(b) Absorption assay

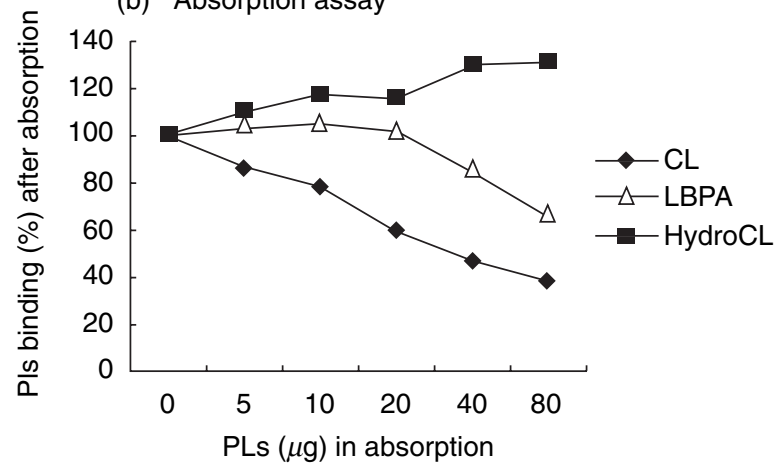

Fig. 2. The specificity of the ELISA was examined by dilution and absorption experiments. (a) A titration curve of two positive reference sera (aLBPA and aCL-positive sera, respectively) was performed by ELISA to show the performance of the tests. IgG binding was dosedependently enhanced by increasing the concentration of phospholipids coated on the ELISA plate wells. Hydrogenated CL derivative (HydroCL) provided a reference structure for basal immunorecognition of the lipid. (b) To further characterize the Ag specificity, absorption tests were performed with three aLBPA/aCL-positive sera by ELISA performed as described in Methods' section. A significant decrease in aLBPA and aCL binding was observed. Each data point represents the mean of triplicate determinations and the figure is representative of all sera tested. 

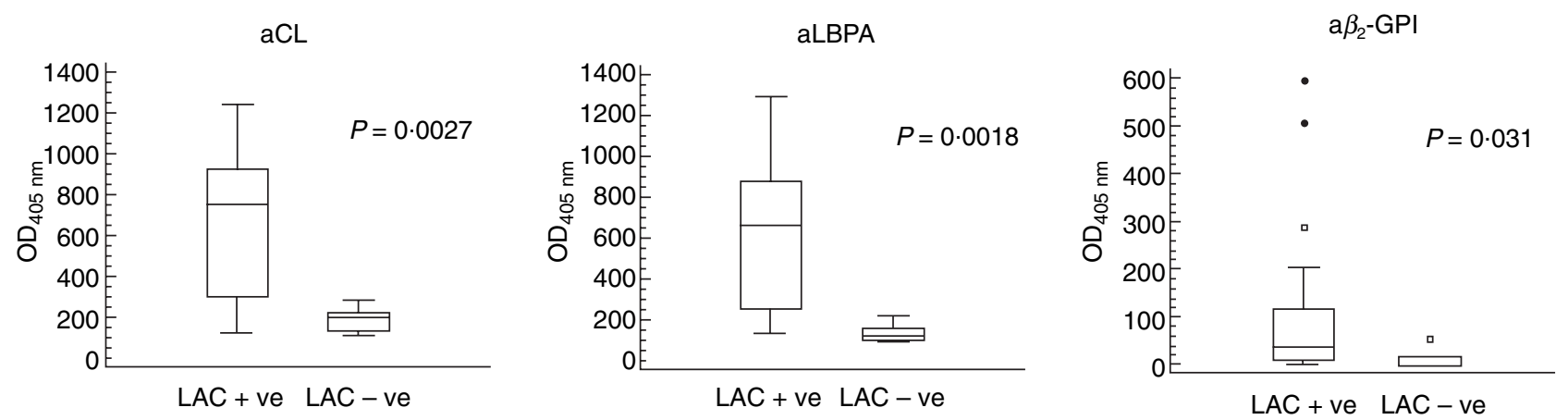

Fig. 3. Box-and-whisker plot of aCL, aLBPA and a $\beta_{2}$-GPI autoantibodies detected by ELISA, in patients grouped according to LAC positivity deduced in each case from recent clinical records. Median, quartiles, range and possibly extreme values are shown. A significantly increased prevalence of aCL, $\mathrm{a} \beta_{2}$-GPI and aLBPA IgG in patients who presented LAC positivity was observed $(P=0 \cdot 0027, P=0.031$ and $P=0 \cdot 0018$, respectively).

tivity ( $P=0.0027$ and $P=0.031$, respectively; Fig. 3). A striking association between LAC positivity and aLBPA was also detected $(P=0.0018$; Fig. 3$)$. On the contrary, we did not find any significant association among the presence of these autoantibodies and each of the clinical features of APS when considered separately (i.e. previous arterial or venous thrombosis, recurrent thrombosis or fetal loss). Nevertheless, all the autoantibodies tested were greatly more prevalent in primary and secondary APS compared to SLE and controls and are thus seromarkers of APS.

\section{${ }_{a} \boldsymbol{\beta}_{2}$-GPI IgG ELISA}

The prevalence of a $\beta_{2}$-GPI IgG was $56 \cdot 6 \%$ (17 of 30 ) in APS and $6.9 \%$ (three of 43 ) in SLE while none of the control sera resulted positive for a $\beta_{2}$-GPI, sensitivity $(95 \%$ CI $) 0.57$ (0.49-0.65), specificity (95\% CI) $0.97 \quad(0.95-1 \cdot 00)$. As reported previously [15], we found a significant positive correlation between a $\beta_{2}$-GPI, aCL and aLBPA IgG reactivity (Fig. 4). According to the prevalence of aCL and a $\beta_{2}$-GPI IgG in APS patients, concordance between the two antibodies was $62.5 \%(15 / 24)$ while concordance between aLBPA and a $\beta_{2}$-GPI IgG was $88 \cdot 2 \%$ (15/17), confirming that aCL, aLBPA and $a \beta_{2}$-GPI represent populations of autoantibodies largely but not completely overlapping.

\section{$\boldsymbol{\beta}_{2}$-GPI dependency of aLBPA and aCL reactivity}

Because it is known that CL and LBPA are able to complex with $\beta_{2}$-GPI [15], but it is also possible to detect serum aCL and aLBPA in the absence of a $\beta_{2}$-GPI in APS patients, we investigated whether aCL and aLBPA antibodies from APS could bind to lipids alone. For this purpose we used two different approaches: ELISA, both in the presence or in the absence (i.e. gelatine) of serum-derived co-factor (blocking buffer, PBS-gelatine) and TLC immunostaining. Interestingly, a reduction, but not reaching a significant difference, was observed in mean reactivity of aLBPA and aCL IgGpositive sera regarding to the presence of $\beta_{2}$-GPI in the
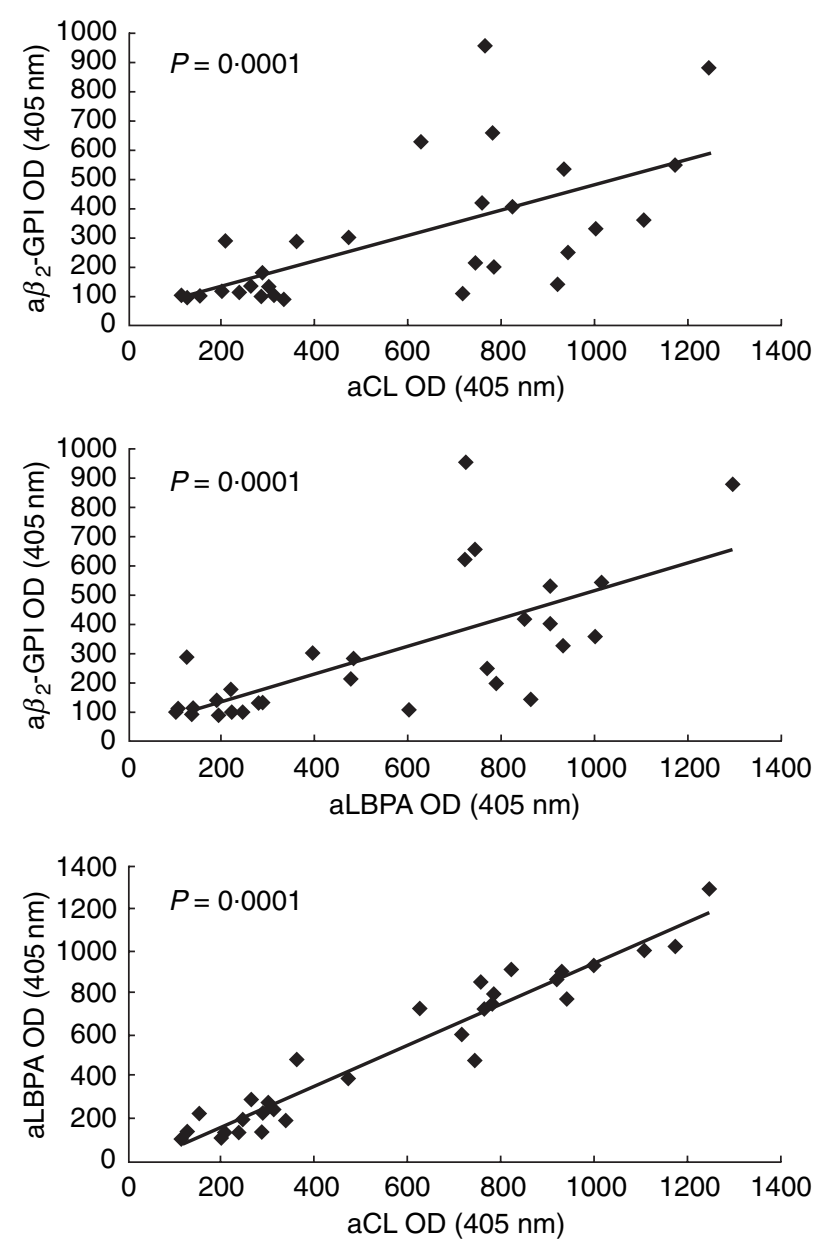

Fig. 4. Correlation analysis between a $\beta_{2}$-GPI, aCL and aLBPA IgG ELISA reactivity in the patients studied was carried out by the Spearman test. As expected, we found a significant correlation between aCL, a $\beta_{2}-$ GPI and aLBPA IgG ELISA reactivity.

ELISA (Fig. 5a). On the other hand, $\beta_{2}$-GPI binding to LBPA was comparable to that of $\mathrm{CL}$ in solid phase (Fig. 5b). Moreover, the partial $\beta_{2}$-GPI independence of aLBPA/aCL-positive sera was confirmed by TLC immunostaining which, in 
(a)

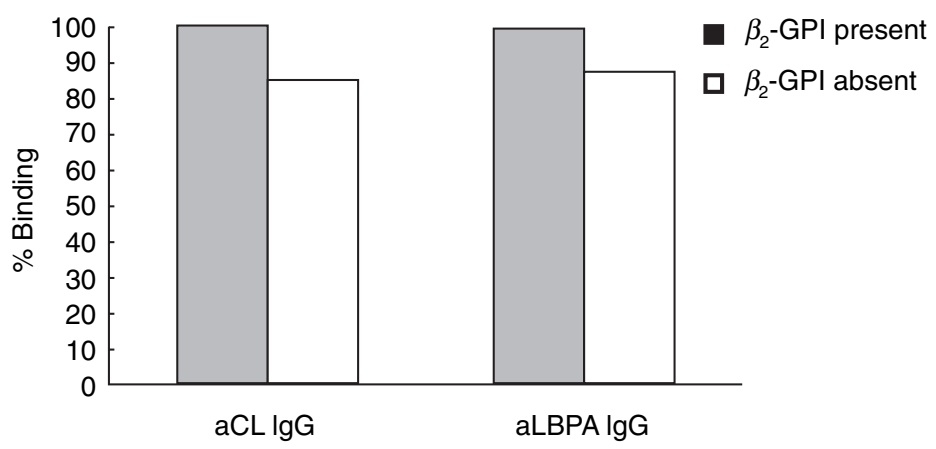

(c)

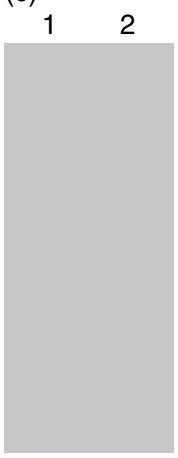

CL LBPA

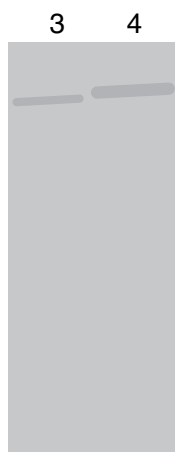

CL LBPA (b)

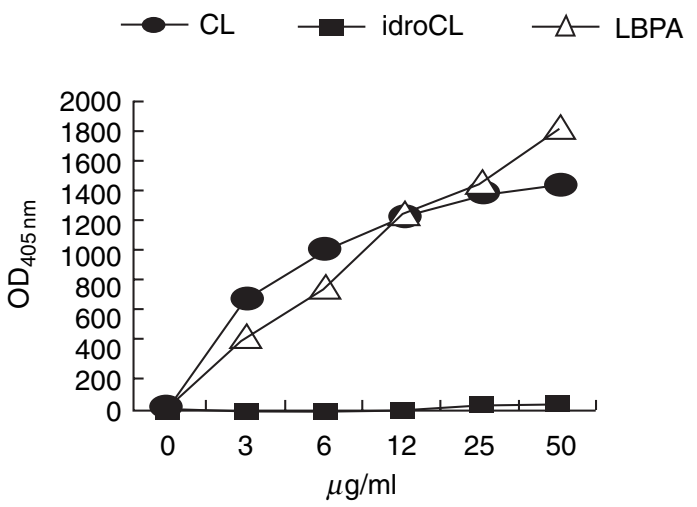

Fig. 5. $\beta_{2}$-GPI dependency of aLBPA and aCL reactivity. (a) aLBPA and aCL IgG ELISA mean reactivity according to the presence of $\beta_{2}$-GPI. The blocking and the washing steps were performed with FCS (10\%) in PBS-Tween 20 to provide the $\beta_{2}$-GPI, or with gelatine $(0 \cdot 25 \%)$, to avoid the presence of $\beta_{2}$-GPI which is commonly associated with FCS. No significant difference of aLBPA and aCL reactivity was observed with different blocking solutions. (b) $\beta_{2}$-GPI binding to LBPA and CL in solid phase. Plates were coated with an increasing amount of lipids and ELISA was performed as described in methods. The plates were treated with PBS-10\% FCS, containing $\beta_{2}$-GPI, for $2 \mathrm{~h}$ at room temperature, and a rabbit polyclonal anti $\beta_{2}$-GPI was used to detect the $\beta_{2}$-GPI protein. Each data point represents the mean of triplicate determinations. $\beta_{2}$-GPI protein could bind to LBPA with comparable or slightly higher affinity than CL. No binding of $\beta_{2}$-GPI to hydrogenated CL (HydroCL) was observed. (c) Immunostaining on TLC plates for aLBPA and aCL. Representative TLC immunostaining of a normal serum (lanes 1 and 2) and of an APS serum positive for both aLBPA and aCL (lanes 3 and 4).

$\beta_{2}$-GPI-free conditions, showed a specific reactivity to CL and LBPA in several representative sera (Fig. 5c).

\section{Discussion}

This study provides evidence that a high percentage of sera from patients with APS show immunoreactivity in a solid phase assay towards LBPA, an anionic phospholipid present in the late endosomes. In addition, a strict correlation between the serum aLBPA and aCL was observed. The HPTLC analysis of the two phospholipid preparations ruled out the possibility that the significant correlation was due to cross-contamination between the two phospholipid samples. Thus, our results show that most sera that bind to cardiolipin also bind to LBPA.

To verify whether the protein co-factor $\beta_{2}$-GPI may mediate the binding of antibodies to LBPA, we compared aLBPA to $a \beta_{2}$-GPI and a significant association was observed, although to a lesser extent than that between aCL and aLBPA. These findings are in agreement with those by Galvede Rochemonteix and colleagues [15], who previously reported the reactivity to LBPA of sera from patients with APS. Nevertheless, we clearly demonstrated by means of solid phase ELISA in $\beta_{2}$-GPI-free conditions and TLC that a population of aCL and aLBPA also can bind directly to lipids in the absence of $\beta_{2}$-GPI. These findings confirm that at least in autoimmune diseases such as APS and SLE, aPL reactivity reveals high antibody heterogeneity [23-25].

To address the diagnostic potential of aLBPA in identifying APS patients, in the present study we further analysed the presence of these antibodies in sera of SLE patients without clinical manifestations of APS, in chronic HCV hepatitis, an infectious disease in which aCL have been previously detected $[26,27]$ and in healthy controls. Our results revealed a highly significant difference in the occurrence of aLBPA antibodies comparing patients with APS and SLE to chronic 
HCV infection or healthy subjects. The overall sensitivity and specificity of aLBPA for APS in our study showed values almost identical to those observed using the $a \beta_{2}$-GPI test, with a very high specificity $(>97 \%)$ but a rather lower sensitivity compared to aCL ( $\sim 55 \%$ versus $80 \%)$. Our results confirmed the recent observation that aCL are present in about $25 \%$ of APS patients fulfilling the Sapporo clinical criteria who are negative for $a \beta_{2}$-GPI or LAC [28]. Although our results suggest that anti-LBPA may not represent an improvement in testing for APS in comparison with anti-CL and $a \beta_{2}$-GPI, this is the first study to demonstrate that antiLBPA are present in a large number of sera of APS patients but virtually absent in SLE or controls, showing almost identical sensitivity and specificity for APS compared to a $\beta_{2}$-GPI (and are slightly more specific although less sensitive than $\mathrm{aCL}$ ) and close association with LAC.

The possibility that aLBPA antibodies may be considered as a novel and reliable serum marker of APS prompted us to hypothesize that these antibodies may also represent a useful tool for clinical monitoring the disease and/or may be related to the clinical features of the syndrome. Thus, we first evaluated the relationship between the presence of aLBPA antibodies and LAC positivity and a very significant association was found. This finding strongly supports the previous observation that aLBPA antibodies display dose-dependent anticoagulant properties in vitro, as they were shown to induce a prolongation of both activated partial thromboplastin time and dilute Russell's viper venom time [14]. This effect was shown to be highly specific and significantly higher than that due to $\beta_{2}$-GPI antibodies alone. We then correlated aLBPA, aCL and $\beta_{2}$-GPI positivity with the main clinical features of the APS syndrome separately, such as arterial and venous thrombosis or fetal loss, but no significant association was found. Nevertheless, this finding is not surprising, because the association of aPL and antico-factor antibodies with clinical manifestation in APS has been long debated (reviewed in [29]). Interestingly, patients with low titres of aCL may have a significant disease and the aCL titre may also fluctuate into normal range at the time of thrombosis [30]. Finally, factors associated with higher risk of thrombosis include a previous history of thrombosis $[31,32]$ and LAC positivity [33]. Longitudinal studies are currently under investigation to clarify the possible predictive value of these antibodies regarding the characteristic clinical features of the syndrome. Interestingly, preliminary results revealed the presence of aLBPA antibodies in two of five sera from patients with the clinical features of the syndrome, but without detectable titres of aCL or a $\beta_{2}$-GPI (data not shown).

Remarkably, our findings demonstrate that LBPA may be an important lipid target for aPL antibodies with potential implications in the pathogenesis of APS. It remains unclear how an antigen which is normally confined in late endosomes becomes a target for these antibodies. Although we cannot exclude the possibility that antibodies may enter target cells [34], the recent demonstration of LBPA in exosomes
[35] shed by the cell supports the view that LBPA, possibly complexed with protein co-factors in these small vesicles, may be the direct target of the antibodies [36]. Alternatively, apoptosis may represent a possible trigger for LBPA exposure on the cell surface. This hypothesis is supported by our recent observation that cardiolipin and its metabolites move to other cellular membranes during death receptor-mediated apoptosis [37].

In conclusion, aLBPA antibodies appear to represent a marker of APS with similar sensitivity and specificity compared to $a \beta_{2}$-GPI and display a slightly higher specificity despite a lower sensibility compared to aCL antibodies. In addition, aLBPA are strongly associated with the presence of LAC, suggesting their pathogenic potential. However, the assessment of their clinical utility as diagnostic and predictive tools as a single test or in combination with aCL and/or $\mathrm{a} \beta_{2}$-GPI in patients with APS deserves further investigation in longitudinal studies.

\section{References}

1 Hughes GR. The anticardiolipin syndrome. Clin Exp Rheumatol 1985; 3:285-6.

2 Wilson WA, Gharavi AE, Koike $\mathrm{T}$ et al. International consensus statement on preliminary classification criteria for definite antiphospholipid syndrome. Arthritis Rheum 1999; 42:1309-11.

3 Galli M, Comfurius P, Maassen C et al. Anticardiolipin antibodies (ACA) directed not to cardiolipin but to a plasma protein cofactor. Lancet 1990; 355:1544-7.

4 McNeil HP, Simpson RJ, Chesterman CN, Krilis SA. Anti-phospholipid antibodies are directed against a complex antigen that includes a lipid-binding inhibitor of coagulation: $\beta_{2}$-glycoprotein I (apolipoprotein H). Proc Natl Acad Sci USA 1990; 87:4120-4.

5 Matsuura E, Igarashi Y, Fujimoto M, Ichikawa K, Koike T. Anticardiolipin cofactor(s) and differential diagnosis of autoimmune disease. Lancet 1990; 336:177-8.

6 Arvieux J, Darnige L, Caron C, Reber G, Bensa JC, Colomb MG. Development of an ELISA for autoantibodies to prothrombin showing their prevalence in patients with lupus anticoagulants. Thromb Haemost 1995; 74:1120-5.

7 Sorice M, Griggi T, Circella A et al. Protein S antibodies in acquired protein S deficiencies. Blood 1994; 83:2383-4.

8 Oosting JD, Derksen RH, Bobbink IW, Hackeng TM, Bouma BN, de Groot PG. Antiphospholipid antibodies directed against a combination of phospholipids with prothrombin, protein C, or protein S. an explanation for their pathogenic mechanism? Blood 1993 81:2618-25.

9 Kaburaki J, Kuwana M, Yamamoto M, Kawai S, Ikeda Y. Clinical significance of anti-annexin $\mathrm{V}$ antibodies in patients with systemic lupus erythematosus. Am J Hematol 1997; 54:209-13.

10 Sorice M, Pittoni V, Griggi T et al. Specificity of anti-phospholipid antibodies in infectious mononucleosis: a role for anti-cofactor protein antibodies. Clin Exp Immunol 2000; 120:301-6.

11 Kobayashi T, Stang E, Fang KS, de Moerloose P, Parton RG, Gruenberg J. A lipid associated with the antiphospholipid syndrome regulates endosome structure and function. Nature 1998; 392:193-7.

12 Gruenberg J, Kreis TE. Membranes and sorting. Curr Opin Cell Biol 1995; 7:519-22. 
13 Schmid SL, Cullis PR. Membrane sorting. Endosome marker is fat not fiction. Nature 1998; 392:135-6.

14 Sorice M, Ferro D, Misasi R et al. Evidence for anticoagulant activity and beta2-GPI accumulation in late endosomes of endothelial cells induced by anti-LBPA antibodies. Thromb Haemost 2002; 87:735-41.

15 Galve-de Rochemonteix B, Kobayashi $\mathrm{T}$ et al. Interaction of antiphospholipid antibodies with late endosomes of human endothelial cells. Arterioscler Thromb Vasc Biol 2000; 20:563-74.

16 Hochberg MC. Updating the American College of Rheumatology revised criteria for the classification of systemic lupus erythematosus. Arthritis Rheum 1997; 40:1725.

17 Fischer K, Chatterjee D, Torrelles J, Brennan PJ, Kaufmann SH, Schaible UE. Mycobacterial lysocardiolipin is exported from phagosomes upon cleavage of cardiolipin by a macrophage-derived lysosomal phospholipase A2. J Immunol 2001; 167:2187-92.

18 Lowry OH, Rosebrough NJ, Farr AL, Randall RJ. Protein measurement with folin phenol reagent. J Biol Chem 1951; 193:26575 .

19 Horkko S, Olee T, Mo L et al. Anticardiolipin antibodies from patients with the antiphospholipid antibody syndrome recognize epitopes in both beta (2)-glycoprotein 1 and oxidized low-density lipoprotein. Circulation 2001; 103:941-6.

20 Horkko S, Miller E, Branch DW, Palinski W, Witztum JL. The epitopes for some antiphospholipid antibodies are adducts of oxidized phospholipid and beta2 glycoprotein 1 (and other proteins). Proc Natl Acad SciUSA 1997; 94:10356-61.

21 Sorice M, Griggi T, Circella A et al. Detection of antiphospholipid antibodies by immunostaining on thin layer chromatography plates. J Immunol Meth 1994; 173:49-54.

22 Brandt JT, Triplett DA, Alving B, Scharrer I. Criteria for the diagnosis of lupus anticoagulants: an update. On behalf of the Subcommittee on Lupus Anticoagulant/Antiphospholipid Antibody of the Scientific and Standardization Committee of the ISTH. Thromb Haemost 1995; 74:1185-90.

23 Sorice M, Circella A, Griggi T et al. Anticardiolipin and anti-beta 2GPI are two distinct populations of autoantibodies. Thromb Haemost 1996; 75:303-8.

24 Zhu M, Olee T, Le DT et al. Characterization of IgG monoclonal anti-cardiolipin/anti-beta2GP1 antibodies from two patients with antipospholipid syndrome reveals three species of antibodies. Br J Haematol 1999; 105:102-9.

25 Lieby P, Soley A, Levallois $\mathrm{H}$ et al. The clonal analysis of anticardiolipin antibodies in a single patient with primary antiphospho- lipid syndrome reveals an extreme antibody heterogenicity. Blood 2001; 97:3820-8.

26 Prieto J, Yuste JR, Beloqui $\mathrm{O}$ et al. Anticardiolipin antibodies in chronic hepatitis C: implication of hepatitis $\mathrm{C}$ virus as the cause of the antiphospholipid syndrome. Hepatology 1996; 23:199-204.

27 Ordi-Ros J, Villarreal J, Monegal F, Sauleda S, Esteban I, Vilardell M. Anticardiolipin antibodies in patients with chronic hepatitis $\mathrm{C}$ virus infection: characterization in relation to antiphospholipid syndrome. Clin Diagn Lab Immunol 2000; 7:241-4.

28 Nash MJ, Camilleri RS, Kunka S, Mackie IJ, Machin SJ, Cohen H. The anticardiolipin assay is required for sensitive screening for antiphospholipid antibodies. J Thromb Haemost 2004; 2:1077-81.

29 Reddel SW, Krilis SA. Testing for and clinical significance of anticardiolipin antibodies. Clin Diagn Lab Immunol 1999; 6:775-82.

30 Drenkard C, Sanchez-Guerrero J, Alarcon-Segovia D. Fall in antiphospholipid antibody at time of thromboocclusive episodes in systemic lupus erythematosus. J Rheumatol 1989; 16:614-17.

31 Finazzi G, Brancaccio V, Moia M et al. Natural history and risk factors for thrombosis in 360 patients with antiphospholipid antibodies: a four-year prospective study from the Italian Registry. Am J Med 1996; 100:530-6.

32 Schulman S, Svenungsson E, Granqvist S. Anticardiolipin antibodies predict early recurrence of thromboembolism and death among patients with venous thromboembolism following anticoagulant therapy. Duration of Anticoagulation Study Group. Am J Med 1998; 104:332-8.

33 Wahl DG, Guillemin F, de Maistre E, Perret C, Lecompte T, Thibaut G. Risk for venous thrombosis related to antiphospholipid antibodies in systemic lupus erythematosus - a meta-analysis. Lupus 1997; 6:467-73.

34 Cheng HM. Cell penetration by antiphospholipid autoantibodies. Immunol Today 1998; 19:49.

35 Laulagnier K, Motta C, Hamdi S et al. Mast cell- and dendritic cellderived exosomes display a specific lipid composition and an unusual membrane organization. Biochem J 2004; 380:161-71.

36 Stinton LM, Eystathioy T, Selak S, Chan EK, Fritzler MJ. Autoantibodies to protein transport and messenger RNA processing pathways: endosomes, lysosomes, Golgi complex, proteasomes, assemblyosomes, exosomes, and GW bodies. Clin Immunol 2004; 110:30-44.

37 Sorice M, Circella A, Cristea IM et al. Cardiolipin and its metabolites move from mitochondria to other cellular membranes during death receptor-mediated apoptosis. Cell Death Diff 2004; 11:113345 . 